\title{
Evaluation of Micronozzle Performance through DSMC, Navier-Stokes and Coupled DSMC/Navier-Stokes Approaches
}

\author{
Federico La Torre ${ }^{1}$, Sasa Kenjeres ${ }^{1}$, Chris R. Kleijn ${ }^{1}$, and Jean-Luc P. A. Moerel ${ }^{2}$ \\ ${ }^{1}$ Department of Multi Scale Physics, Faculty of Applied Sciences, Delft University of \\ Technology, \\ Prins Bernhardlaan 6, 2628 BW Delft, The Netherlands \\ F.LaTorre@tudelft.nl, S.Kenjeres@tudelft.nl, \\ C.R.Kleijnatudelft.nl \\ ${ }^{2}$ Department of System Performance and Survivability, TNO Defence, Security and Safety, \\ P.O.Box 45,2280 AA Rijswijk, The Netherlands \\ Jean-Luc.Moerel@tno.nl
}

\begin{abstract}
Both the particle based Direct Simulation Monte Carlo (DSMC) method and a compressible Navier-Stokes based continuum method are used to investigate the flow inside micronozzles and to predict the performance of such devices. For the Navier-Stokes approach, both slip and no-slip boundary conditions are applied. Moreover, the two methods have been coupled to be used together in a hybrid particle-continuum approach: the continuum domain was then investigated by solving the Navier-Stokes equations with slip wall boundary condition, whereas the region of rarefied regime was studied by DSMC. The section where the domain was split was shown to have a great influence in the prediction of the nozzle performance.
\end{abstract}

Keywords: Direct Simulation Monte Carlo, Coupled Method, Rarefied Gas Flow, Slip Regime, Micronozzle.

\section{Introduction}

Various trends in the spacecraft industry are driving the development of so-called micro-satellites (which have a typical volume of $1 \mathrm{dm}^{3}$ ). For missions that require a satellite propulsion system, the existing propulsion systems are too large and too heavy. To address this problem, in The Netherlands a large multidisciplinary research program is being carried out [1] aimed at the development of micro-propulsion systems with a typical thrust in the order of $\mathrm{mN}$. Such propulsion systems can be used to maintain or adjust the orbit of space micro-satellites or to provide long duration low thrust acceleration.

One of the simplest forms of micropropulsion is the cold gas thruster: the gas, pressurized in a microtank, is accelerated and expanded through a convergentdivergent nozzle. The typical dimensions of the throat of the nozzles are below one millimeter. Due to these small characteristic dimensions, there are some important differences in the gas flow field in such a micro-nozzle, compared to conventional large scale nozzles. The increase of the surface-to-volume ratio and the reduction of 
the Reynolds number lead to the presence of large viscous losses and to the formation of thicker boundary layers (relative to the geometrical dimensions), with a consequent lower efficiency of such systems. Also the effect of surface roughness, with a characteristic length comparable to the throat diameter, cannot be neglected. These aspects of micro-thruster flow have been investigated in [2].

Another important aspect of micro-nozzle gas flow, especially when going even further down in thrust to the $\mu \mathrm{N}$ range, is the effect of rarefaction. At these low thrusts and small nozzle dimensions, the throat diameter and gas flow boundary layer thickness become comparable to mean free path of the molecules and we enter a different gas flow regime. An important parameter in this respect is the Knudsen number, defined as the ratio between the mean free path of the gas molecules $\lambda$, and a characteristic length $\mathrm{L}$ of the flow:

$$
\mathrm{Kn}=\frac{\lambda}{\mathrm{L}}
$$

When $\mathrm{Kn}>10$, molecule-molecule interactions are negligible compared to molecule-wall interactions, and the flow is in the so-called free molecular regime. This regime can be accurately and efficiently modeled through free-flight (ballistic) molecular models. When $\mathrm{Kn}<0.1$, the flow is dominated by intermolecular interactions and can be described by continuum equations, such as the Navier-Stokes equations. However, for $0.1>\mathrm{Kn}>0.01$ important deviations from the Navier-Stokes solutions occur in the vicinity of solid walls. This is the so-called slip flow regime, requiring the use of modified boundary conditions for momentum and energy transfer at the walls. For $10>\mathrm{Kn}>0.1$, molecule-molecule and molecule-wall interactions are equally important, and the flow is in the so-called transition regime. In this regime, neither continuum models nor free molecular models can be applied.

When operated in space, the gas flow in micro-nozzles experiences all regimes mentioned above, from the continuum regime (in the gas chamber and the convergent part of the nozzle), to slip flow and transition regimes (in the divergent part of the nozzle), up to the free molecular regime (far from the exit of the nozzle). The need to accurately and consistently model all these regimes is a challenge from a computational point of view.

Some attempts have been made to use Navier-Stokes equations with slip wall boundary conditions, for example in [3] [4] [5] [6]. However, such an approach is not accurate when the Knudsen number exceeds 0.1, see e.g. [16] [17]. In the transition regime, the most accurate and flexible method to use is Direct Simulation Monte Carlo (DSMC) [22]. It is more efficient than deterministic molecular models such as Molecular Dynamics (MD), and more flexible and generally applicable than methods based on direct solution of the Boltzmann equations. Full DSMC simulations, for different micronozzle configurations were performed in [7] [8] [9] [10].

In principle, DSMC is valid for all flow regimes. However, in order for its solutions to be accurate the requirements on number of modeled particles, grid size and time step size increase severely with decreasing Knudsen number. As a result, the computational expenses of a proper DSMC simulation scale with $\mathrm{Kn}^{-4}$. In practice it is therefore not possible to use DSMC in all flow regions, and the application of DSMC to an entire micro-nozzle flow cannot be expected to be accurate in the low $\mathrm{Kn}$ number zones. 
In order to overcome this problem, a hybrid Navier-Stokes / DSMC model can be considered. The method applies the continuum approach in low Kn regions, while DSMC is applied in regions where rarefaction effects need to be considered. Some of these hybrid models were proposed in [11] [12] [13] [14] [15].

In the present paper, the performance of a $\mu \mathrm{N}$ thrust micro-nozzle is evaluated through different numerical models, i.e. Navier-Stokes equations with slip flow boundary conditions, full DSMC, and coupled Navier-Stokes / DSMC. After a brief introduction on the studied problem (Section 2), the coupled hybrid approach will be explained in the Section 3, while the numerical results will be shown in Section 4. Conclusions and some remarks will be addressed in Section 5.

\section{Description of the Problem}

The studied nozzle is shown in Fig. 1. This geometry is typical for micronozzle thrusters as used in practice [1] [2]. Two different regions can be distinguished: a convergent part, where the flow is accelerated from the initial subsonic velocity to the sonic condition (which is reached at the throat), and a divergent part, where the flow expands to the outlet in a supersonic regime.

The analyzed configuration has both convergent and divergent angles equal to $20^{\circ}$, an inlet area equal to 7.27 times the throat area, and an outflow area of 23.23 times the throat area. The throat radius is $3.56 \mu \mathrm{m}$, while a sharp angle is considered to connect the convergent and the divergent. In the inlet, nitrogen gas is introduced at a fixed pressure and temperature of respectively $\mathrm{P}_{0}=3.5$ bar and $\mathrm{T}_{0}=300 \mathrm{~K}$. A vacuum condition is assumed outside. The walls are modeled as isothermal at $\mathrm{T}_{0}=300 \mathrm{~K}$. Under these conditions, the ideal thrust $\mathrm{T}_{\text {ideal }}$ computed from the $1 \mathrm{D}$ isentropic flow theory [18], is about $25 \mu \mathrm{N}$.

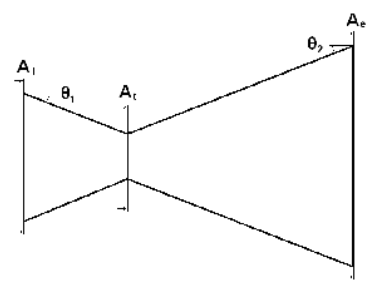

Fig. 1. The nozzle geometry configuration considered in the present study, with the geometric parameters of interest

\section{Numerical Approach}

\subsection{The CFD Solver}

All the CFD simulations presented in this paper have been obtained with the general purpose code Fluent version 6.3 [19]. The studied nozzle has a conical shape and therefore a 2D-axisymmetric flow is considered. The grid, generated with Gambit [20], consists of quadrilateral elements, with 100 grid cells in the radial direction and about 600 grid cells in the axial direction. The nitrogen gas is treated as an ideal gas, 
with temperature dependent viscosity and thermal conductivity computed from kinetic theory. As an initial guess for the solution, the pressure $\mathrm{P}_{\mathrm{e}}$ at the outflow was computed from the 1D isentropic flow theory [18].

The solved equations include the continuity equation, the momentum balance (Navier Stokes) equations in compressible form, and the thermal energy equation. The equations were discretized in space using a second order upwind scheme, and solved using Fluent's explicit coupled pressure based solver. This choice was done in order to enable the option to impose a slip velocity boundary condition at the wall, according to the formulas by Maxwell and von Smoluchowski [21], approximated by the solver as explained in [19].

\subsection{Direct Simulation Monte Carlo}

The DSMC method, as developed originally by G.A. Bird, is comprehensively described in [22]. This method is not based on solving partial differential equation, such as the Navier-Stokes equations, but describes the state of the system by computing the positions and velocities of computational particles, each of which represents an assigned number of particles in the real flow. The computational domain is divided into grid cells, through which the particles can move. All the properties of the particles are stored and updated each time step during the calculation. First, the particles are moved as if they did not interact, and any particles that reach a boundary are processed according to the appropriate boundary condition. Second, after all the particles have moved, a certain number of particles from each grid cell are randomly selected for collisions, according to one of the collisions models [22]. This decoupling between the translational movement of the particles and their collisions is accurate only when the time step is a fraction of the mean collision time. On the other hand, the random selection of the particles as possible candidates to be collision partners leads to the restriction that their mean separation needs to be a fraction of the mean free path. This means that the cell size should be small enough in order to avoid that energy or momentum are transported over long distances in an unrealistically short time. Finally, the number of particles per cell needs to be high enough to avoid repeated collisions between the same particles and make the collision process statistically accurate. All these constraints make DSMC computationally expensive.

The DSMC solver used in the present study has been implemented in the 3dimensional general purpose DSMC code X-Stream [24] and validated for a wide range of problems [25], amongst which the case of a low pressure micronozzle as studied in [9]. In all the simulations presented in this paper, a Variable Soft Sphere (VSS) model has been used, with the corresponding parameter set from the literature [22]. For the interaction between the nitrogen particles and the isothermal silicon walls a thermal accommodation coefficient of 0.5 was assumed, according to the formula provided in [23]. In order to model axial-symmetry, a radial weighting factor has been introduced, as described in [22] [25], such that a molecule located far from the axis represents more real molecules than one near the axis. 


\subsection{The Hybrid CFD / DSMC Approach}

Figure 2 shows a first comparison between the solutions obtained from a full CFD simulation, both with a partial slip (wall accommodation coefficient 0.5 ) and a no-slip wall boundary condition, and the solutions from full DSMC. When assuming partial slip at the wall, the CFD solution matches the DSMC solution much better, particularly in the divergent. However, it cannot be expected that the full DSMC solution accurately represents the 'true' flow physics, since the DSMC computational requirements, as explained above, could not be satisfied in the whole domain, in particular in the convergent part of the nozzle where the pressure is high and the Knudsen number is low.

Because of the above, we will develop a hybrid approach, applying CFD with partial slip in the upstream (convergent) part of the domain, and DSMC in the downstream (divergent) part of the domain.
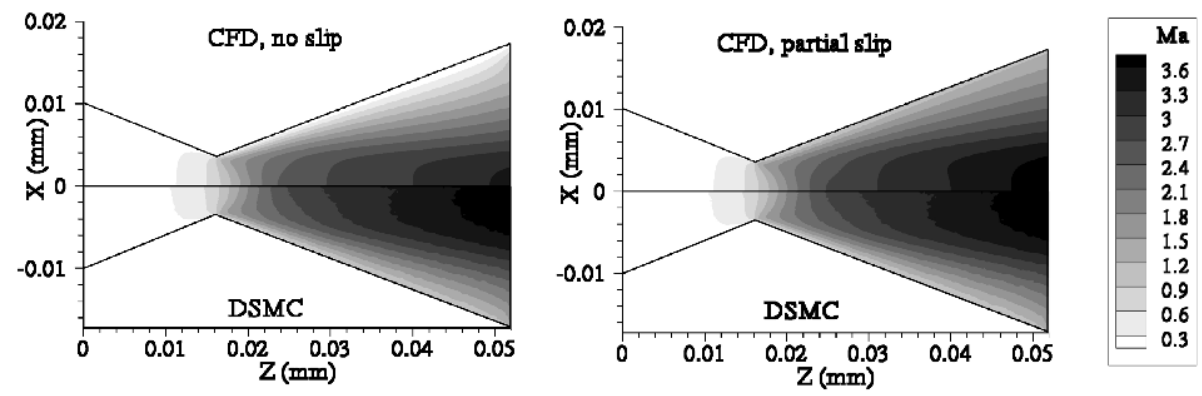

Fig. 2. Comparison of Mach number contours from the solution obtained by full CFD (half upper picture) and full DSMC (half bottom picture). On the left, a no-slip velocity was imposed at the wall for the CFD problem, while on the right a slip flow boundary condition with accomodation coefficient 0.5 was applied.

In the present paper a single step coupling approach between CFD and DSMC has been conducted. First, a CFD simulation is performed for the entire nozzle. From this an estimation of the local Knudsen number throughout the nozzle is made, see Fig. 3. Four different definitions for Kn have been considered, depending on the definition of the characteristic length $\mathrm{L}$ in (1).

If $\mathrm{L}$ is taken to be the throat diameter $\mathrm{D}_{\mathrm{t}}$, (1) becomes:

$$
\mathrm{Kn}=\frac{\lambda}{\mathrm{D}_{\mathrm{t}}}
$$

To account for local variations in characteristic length scales of the flow, a more accurate definition of Kn can be obtained using the gradient length scale of a certain physical quantity:

$$
\mathrm{Kn}_{\mathrm{Q}}=\frac{\lambda}{\mathrm{Q}}|\nabla \mathrm{Q}|
$$


Figure 3 shows Knudsen numbers based on (a) the throat diameter, and gradient length scales of (b) pressure, (c) density and (d) temperature. When the maximum of the four computed values of Kn exceeds a value of approximately 0.1 , the continuum CFD approach is considered to be inaccurate. From Fig. 3 it is clear that this is the case for (a large part of) the divergent.

Therefore, the computational domain was subsequently split between a CFD part upstream, and a DSMC part downstream of a certain vertical division plane. The predicted solution from CFD at this division plane was then applied as inlet boundary condition for a DSMC solution downstream from the division plane. We considered division planes located at the throat, and 6, 12, 18 and $24 \mu \mathrm{m}$ downstream the throat; these sections will be referred, respectively, as Th, L1, L2, L3 and L4.
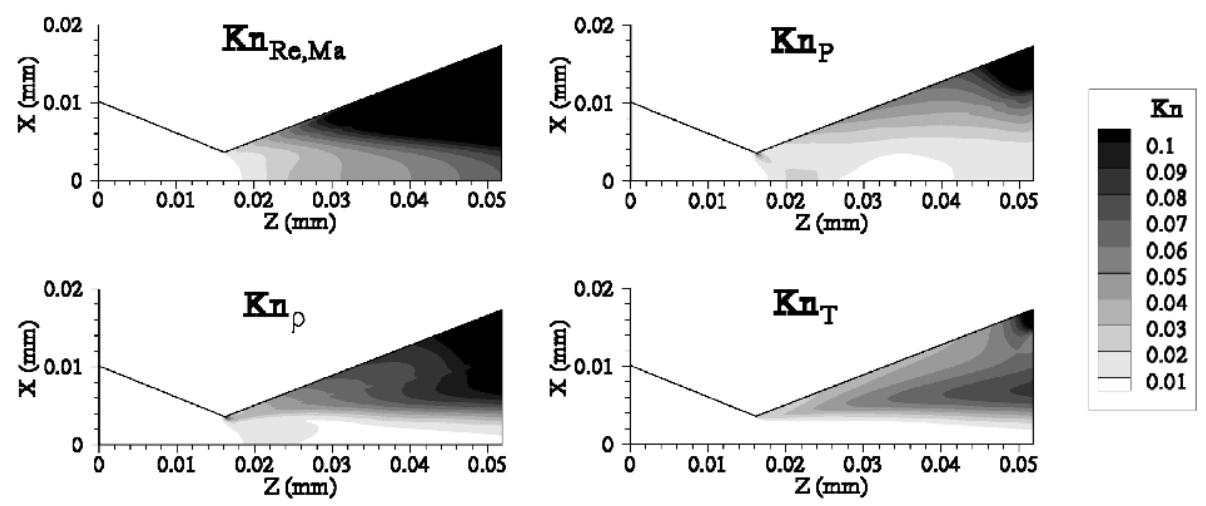

Fig. 3. Knudsen number contours evaluated by (2) (upper left picture), and (3) with pressure (upper right picture), density (bottom left picture) and temperature (bottom right picture) as characteristic quantity, from the solution obtained by full CFD with no slip wall boundary condition

\section{Results}

To compare the full CFD, full DSMC and hybrid CFD/DSMC approaches for modeling the gas flow in the micro nozzle, we will focus on the predicted nozzle performance. This performance is evaluated through two parameters. The first is defined as:

$$
\eta=\frac{T_{\text {real }}}{T_{\text {ideal }}}
$$

where the ideal thrust $\mathrm{T}_{\text {ideal }}$ is given by the $1 \mathrm{D}$ isentropic flow theory, and the real thrust $\mathrm{T}_{\text {real }}$ is computed from

$$
\mathrm{T}_{\text {real }}=\int_{\substack{\text { outlet } \\ \text { area }}}\left[\mathrm{mV}_{\mathrm{e}}+\left(\mathrm{p}_{\mathrm{e}}-\mathrm{p}_{\mathrm{a}}\right)\right] \mathrm{dA}
$$


The subscript e refers to the conditions at the exit section of the nozzle, while the subscript a refers to the ambient conditions outside the nozzle.

The second performance parameter is the specific impulse, defined as:

$$
\mathrm{I}_{\mathrm{sp}}=\frac{\mathrm{T}}{\mathrm{mg}}
$$

which describes the change in momentum of the satellite per unit of propellant weight (on earth), so that the higher it is, the less propellant is needed to gain a certain amount of momentum.

The nozzle performances as computed from the different modeling approaches have been listed in Table 1 .

Table 1. Nozzle performance evaluated by the solutions from different numerical approaches

\begin{tabular}{cccc}
\hline Configuration & $\mathrm{T}, \mathrm{mN}$ & $\eta$ & $\mathrm{I}_{\mathrm{sp}}, \mathrm{s}$ \\
\hline full CFD, no-slip wall & $1.96 \times 10^{-2}$ & 84.52 & 68.44 \\
full CFD, partial slip wall & $2.06 \times 10^{-2}$ & 88.78 & 68.98 \\
full DSMC & $2.00 \times 10^{-2}$ & 86.26 & 66.71 \\
CFD slip, DSMC from Th & $1.99 \times 10^{-2}$ & 86.04 & 66.85 \\
CFD slip, DSMC from L1 & $2.04 \times 10^{-2}$ & 87.83 & 68.02 \\
CFD slip, DSMC from L2 & $2.05 \times 10^{-2}$ & 88.29 & 68.37 \\
CFD slip, DSMC from L3 & $2.06 \times 10^{-2}$ & 88.97 & 68.90 \\
CFD slip, DSMC from L4 & $2.07 \times 10^{-2}$ & 89.33 & 69.17 \\
\hline
\end{tabular}

When a slip wall boundary condition is applied, the full CFD approach predicts a higher performance than the full DSMC approach both in terms of thrust and specific impulse. The presence of slip velocity allows the flow to reach higher velocities at the outflow. In general, as can be noted from Fig. 2, the use of slip flow boundary conditions leads to a decrease of the subsonic part of the boundary layer in the divergent, and to an overall decrease of the thickness of that boundary layer. This leads to a larger expansion of the flow, with a consequent higher velocity and a lower pressure in the outlet. This can be observed through the investigation of the exit profiles for velocity and pressure, as shown in Fig. 5.

On the other hand, a full CFD simulation with no-slip wall boundary condition gives an under-prediction of the nozzle thrust compared to full DSMC. This lower thrust can be related to the presence of a thicker subsonic boundary layer and to a consequent lower mass flow and no velocity close to the wall. In contrast with the underprediction of the thrust, the full CFD simulation with no-slip boundary condition leads to an overprediction of the specific impulse compared to the full DSMC solution, because the mass flow in this case is lower than the one observed when a partial slip wall is modeled. In general, both the full CFD and the hybrid CFD/DSMC solutions led to a higher specific impulse than the full DSMC solution.

From Table 1 it can be seen that the thrusts and specific impulses predicted by the hybrid CFD/DSMC modeling approach, depend on the location of the vertical division plane between the CFD and DSMC regions. When the division plane is located at the throat, the thrust and specific impulse predicted by the hybrid solution are similar 
to those of a full DSMC solution. When the division plane is located far downstream in the divergent, the thrust and specific impulse predicted by the hybrid approach are similar to that of a full CFD solution. This because a DSMC solution started too far downstream from the throat does not properly take into account the whole domain where rarefied effects are present and is not able to simulate the full expansion of the flow, with a consequent too high exit pressure (5). The solution from the hybrid approach, both in terms of efficiency and of the flow field inside the nozzle, approaches that of a full DSMC approach when most of the divergent is solved with DSMC. This is also visible in Fig. 4.
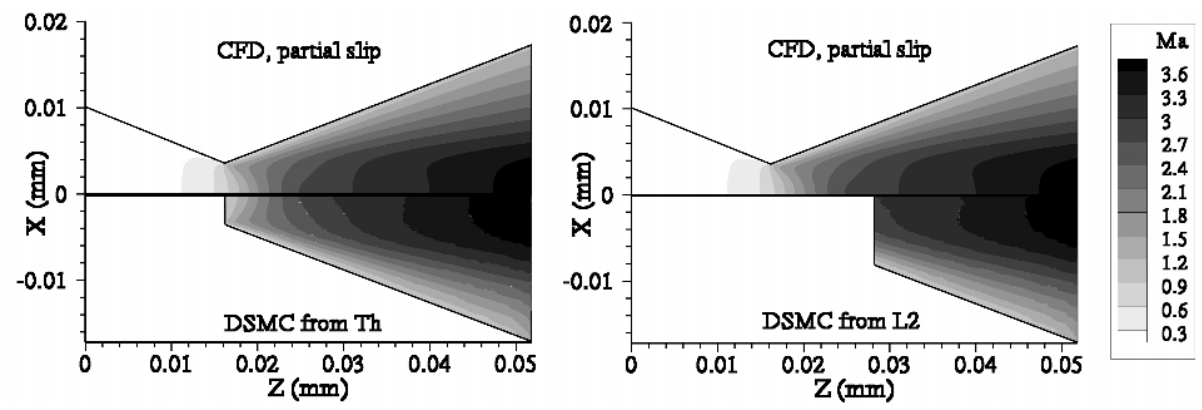

Fig. 4. Comparison of Mach number contours from the solution obtained by full CFD and partial slip wall velocity (half upper picture) and DSMC with inlet boundary conditions extrapolated from the CFD solution (half bottom picture), at the throat (left), section L2 (right)
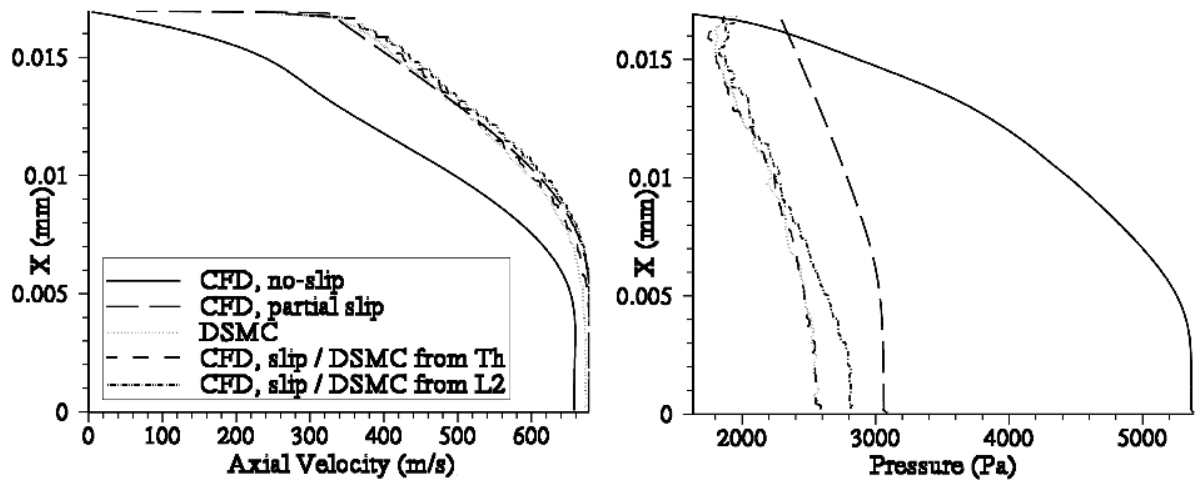

Fig. 5. Profiles for the exit velocity (left) and exit pressure (right) obtained by CFD, DSMC and coupled CFD/DSMC

\section{Conclusions}

A fixed micronozzle configuration allowing a thrust of about $25 \mu \mathrm{N}$ has been studied through CFD, DSMC and coupled CFD/DSMC simulations. The continuum model, both with no-slip and slip flow at the walls, showed different results from DSMC for 
the flow field inside the nozzle in the divergent, especially close to the outflow, where strong rarefied effects start to appear. In general, a better agreement between DSMC and CFD was observed when a slip wall boundary condition was implemented. From this point of view, a fully CFD simulation with slip wall could be considered accurate enough to predict the flow field inside a $25 \mu \mathrm{N}$ thruster.

On the other hand, although the outflow velocity in the expansion core of the nozzle was close to the solution from DSMC, a full CFD solution with slip boundary condition was found to overpredict the thrust compared to a DSMC solution. It was found that this is due to the fact that a CFD solution - even with slip wall boundary conditions - overpredicts the pressure field in the outflow region, which enhances the nozzle performance, both in terms of efficiency and of specific impulse. A static hybrid CFD / DSMC approach confirmed the importance of the pressure term in the calculation of the thrust of the nozzle, and showed that a great part of the divergent needs to be modeled by DSMC to allow the prediction of a full expansion. In this case, the final solution strongly depends on the division plane downstream from which DSMC is applied, and approaches to the full DSMC solution when a larger part of the divergent is solved through DSMC.

Since the present, static and one-way, coupling between CFD and DSMC proved to be rather sensitive to the exact location of the division plane between the two computational regions, there is a need to develop an adaptive, two-way coupling approach, in which the results from the DSMC solution in the downstream part of the nozzle are used to update the boundary conditions for the CFD solution in the upstream part, and an adaptive overlap region is applied to ensure that the CFD and DSMC solutions are consistent with each other. We believe that such an approach can be realized using the coupling between a general purpose CFD code such as Fluent, and a DSMC code such as X-Stream. It should be pointed that numerical issues in the interface between the two methods could also play a role. Since the difference in the performance as predicted by the various approaches is less than a few percents, extra cases with even lower thrusts and nozzle dimensions should be studied to conclusively determine the merits of the various simulation approaches for micro nozzle gas flow. This is object of current investigation.

\section{Acknowledgement}

This project is founded by MicroNed, one of the Dutch BSIK research programs.

\section{References}

1. Moerel, J.L.P.A., Sanders, H.M., Louwerse, M.C., Jansen, H.V., Boscher, J., Zandbergen, B.T.C., La Torre, F., Kenjeres, S., Kleijn, C.R.: Development of Micro Propulsion System Technologies for Minisatellites in The Netherlands. In: Proceedings of the 5th Int. Spacecraft Propulsion Conference (2008)

2. La Torre, F., Kenjeres, S., Kleijn, C.R., Moerel, J.L.P.A., Zandbergen, B.T.C.: Influence of Boundary Layer Formation and Surface Roughness on the Thrust of Micro-Nozzle. In: Proceedings of the 5th Int. Spacecraft Propulsion Conference (2008) 
3. Liu, M., Zhang, X., Zhang, G., Chen, Y.: Study on Micronozzle Flow and Propulsion Performance using DSMC and Continuum Methods. Acta Mechanica Sinica 22, 409-416 (2006)

4. Markelov, G.N., Ivanov, M.S., Ketsdever, A.D., Wadsworth, D.C.: Numerical Study of Cold Gas Micronozzle Flows. In: Proceedings of the 33rd Aerospace Science Meeting and Exhibit, 1999, AIAA 99-0166 (1999)

5. Gadepalli, V.V.V., Lin, C.: Navier-Stokes Modeling of Gas Flows in a De-Laval Micronozzle. In: Proceedings of the 44th AIAA Aerospace Sciences Meeting and Exhibit (2006)

6. Mo, H., Lin, C., Gokaltun, S., Skudarnov, P.V.: Numerical Study of Axisymmetric Gas Flow in Conical Micronozzle by DSMC and Continuum Methods. In: Proceedings of the 44th AIAA Aerospace Sciences Meeting and Exhibit, AIAA 2006-991(2006)

7. Xie, C.: Characteristics of Micronozzle Gas Flows. Journal of Physics of Fluids 19(3) (2007)

8. Markelov, G.N., Ivanov, M.S.: Numerical Study of 2D/3D Micronozzle Flows. In: Proceedings of the 22nd International Rarefied Gas Dynamics Symposium, AIP Conference Proceedings, vol. 585, pp. 539-546 (2001)

9. Buoni, M., Kietz, D., Aslam, K., Subramaniam, V.V.: Simulation of a Compressible Gas Flow in a Mmicronozzle. In: Proceedings of the 35th AIAA Thermophysics Conference, AIAA 2001-3073 (2001)

10. Alexenko, A.A., Levin, D.A., Gimelschin, S.F., Collins, R.J.: Numerical Modeling of Axisymmetric and Three-Dimensional Flows in MEMS Nozzle. AIAA Journal 40(5), 897-904 (2002)

11. Garcia, A., Bell, J.B., Crutchfield, W.Y., Alder, B.J.: Adaptive Mesh and Algorithm Refinement using Direct Simulation Monte Carlo. Journal of Computational Physics 154, 134-155 (1999)

12. Lou, T., Dahlby, D.C., Baganoff, D.: A Numerical Study Comparing Kinetic Flux-Vector Splitting for the Navier-Stokes Equations with a Particle Method. Journal of Computational Physics 145, 489-510 (1998)

13. Le Tallec, P., Mallinger, F.: Coupling Boltzmann and Navier-Stokes Equations by Half Fluxes. Journal of Computational Physics 136 (1997)

14. Wijesinghe, H.S., Hadijconstantinou, N.G.: Discussion of Hybrid Atomistic-Continuum Methods for Multiscale Hydrodynamics. International Journal for Multiscale Computational Engineering 2, 189-202 (2004)

15. Abbate, G., Thijsse, B.J., Kleijn, C.R.: Validation of a Hybrid N-S/DSMC Method for Multi-Scale Transient and Steady-State Gas Flows. International Journal for Multiscale Computational Engineering 6, 1-12 (2008)

16. Cercignani, C.: The Boltzmann Equation and its applications. Springer, Heidelberg (1988)

17. Hadjiconstantinou, N.G.: Comment on Cercignani's Second-Order Slip Coefficient. Physics of Fluids 15, 2352 (2003)

18. Sutton, G.P., Biblarz, O.: Rocket Propulsion Elements. John Wiley \& Sons, Chichester (2001)

19. Fluent 6 Tutorial Guide. Fluent Incorporate (2005)

20. Gambit 2 Tutorial Guide. Fluent Incorporate (2001)

21. Gad-el-Hak, M.: The Fluid Mechanics of Microdevices - the Freeman Scholar Lecture. Journal of Fluids Engineering 121, 5-33 (1999)

22. Bird, G.A.: Molecular Gas Dynamics and the Direct Simulation of Gas Flows. Oxford Science Publications, Oxford (1994)

23. Goodman, F.O., Wachman, H.Y.: Formula for thermal accommodation coefficients. Journal of Chemical Physics 46(6), 2376-2386 (1967)

24. CVD-X User Manual. TNO (2007)

25. Dorsman, R.: Numerical Simulations of Rarefied Gas Flows in Thin Film Processes. PhD thesis, Delft (2007) 